\title{
Chemical Equilibrium in Heavy Ion Collisions: Rapidity Dependence.
}

\author{
F. Becattini ${ }^{1}$, J. Cleymans ${ }^{2}$ \\ ${ }^{1}$ 1, Università di Firenze and INFN Sezione di Firenze \\ Largo E. Fermi 2, I-50125, Florence, Italy. \\ ${ }^{2}$,UCT-CERN Research Centre and Department of Physics, University of Cape \\ Town, ZA-7701 Rondebosch, South Africa \\ E-mail: becattini@fi.infn.it Jean.Cleymans@uct.ac.za
}

\begin{abstract}
Particle yields in heavy ion collisions show an overwhelming evidence for chemical or relative chemical equilibrium at all beam energies. The rapidity dependence of the thermal parameters $T$ and $\mu_{B}$ can now be determined over a wide range of rapidities and show a systematic behavior towards an increase in $\mu_{B}$ away from mid-rapidity.
\end{abstract}

\section{Introduction}

Over the past decade, the analysis of particle multiplicities in heavy ion collions has shown overwhelming evidence for chemical equilibrium in the final state except for particles carrying strangeness which are mildly suppressed; however, their relative yields fulfill statistical equilibrium. A summary as of 2006, combining the results from many different groups [1], is shown in Fig. 1. Except for particle multiplicities at RHIC

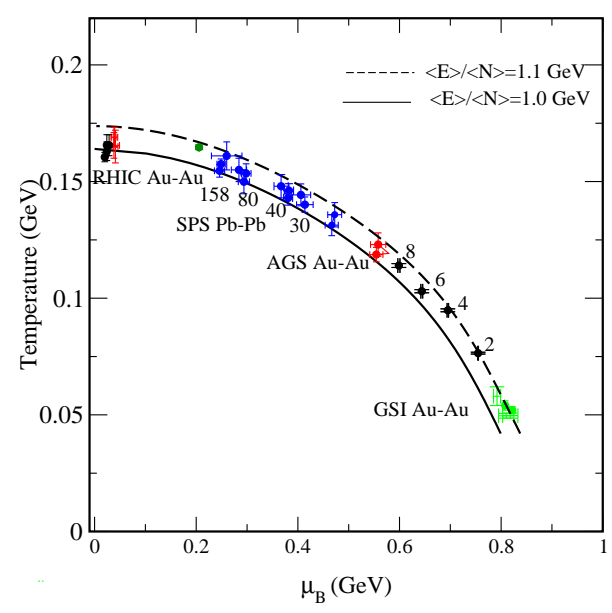

Figure 1. Temperature vs. $\mu_{B}$ us determined from heavy ion collisions at different beam energies. The lower AGS points are based on a preliminary analysis of $4 \pi$ data. 
energies, all data in Fig. 1 use integrated particle yields, the very systematic change of thermal parameters over the full range of beam energies is one of the most impressive features of relativistic ion collisions to date. It is now possible to use the thermal model to make solid predictions for particle multiplicities at LHC energies [2] and to determine which beam energy will lead to the highest baryon density at freeze-out [3]. With chemical equilibrium for integrated yields thus firmly established, we focus on other properties, in particular, since the rapidity distributions of identified particles is now becoming available also at RHIC energies [7], it is now possible to determine the rapidity dependence of thermal parameters. A first analysis was done by Stiles and Murray [4] for the data obtained by the BRAHMS collaboration at $200 \mathrm{GeV}$ [6, 7]. This shows a clear dependence of the baryon chemical potential on rapidity in particular due to the changing $\bar{p} / p$ ratio. Subsequently it was shown by Röhrich [5] that the particle ratios at large rapidities are consistent with those measured at the SPS energies. This opens the possibility to compare measurements for e.g. the $K^{+} / \pi^{+}$ratio at high rapidities and check them with the corresponding values measured in the energy scan at the SPS, thus complementing the rapid variation of this ratio as a function of beam energy. A further analysis of the rapidity dependence was recently done in Ref. [8]. A detailed report of our results will be presented elsewhere [9].

\section{Rapidity Distribution}

The general procedure is as follows: the rapidity axis is populated with fireballs following a gaussian distribution function given by $\rho\left(y_{F B}\right)$ where $y_{F B}$ is the rapidity of the fireball. Particles will appear when the fireball freezes out and will follow a thermal distribution centered around the position of the fireball

$$
\rho\left(y_{F B}\right)=\frac{1}{\sqrt{2} \pi \sigma} \exp \left(-\frac{y_{F B}^{2}}{2 \sigma^{2}}\right) .
$$

The momentum distribution of hadron $i$ is then calculated from the distribution of fireballs as given by Eq. 11 along the rapidity axis as follows

$$
E_{i} \frac{d^{3} N_{i}}{d^{3} p}=\int_{-\infty}^{\infty} \rho\left(y_{F B}\right) E_{i} \frac{d^{3} N_{1}^{i}}{d^{3} p}\left(y-y_{F B}\right) d y_{F B}
$$

where $E_{i} \frac{d^{3} N_{1}^{i}}{d^{3} p}$ is the the distribution of hadrons from a single fireball. The temperature $T$ and the baryon chemical potential $\mu_{B}$ will depend on the rapidity of the fireball and are not assumed to be constant.

An important parameter is the width of the distribution. For the RHIC data at $200 \mathrm{GeV}$ this was determined from the $\pi^{+}$'s as these are very sensitive to the value of $\sigma$ and less to variations in the baryon chemical potential. The width of the distribution $\sigma=2.25$ is compatible with the values quoted by the BRAHMS collaborationi [6], e.g. $\sigma_{\pi^{+}}=2.25 \pm 0.02$ and $\sigma_{\pi^{-}}=2.29 \pm 0.02$. The hadrons described by eq. [2] are mainly resonances. Only a fraction of these are stable under strong interactions. The majority of them will decay into stable hadrons at chemical freeze-out, hence the need to implement multi-particle decays. 


\section{Freeze-Out Curve}

We assume that the temperature $T$ and the chemical potential $\mu_{B}$ are always related via the freeze-out curve as given in Fig. [1]; if the temperature varies along the rapidity axis, then also the chemical potential will vary. Thus a decrease in the temperature of the fireball will be accompanied by an increase in the baryon chemical potential. In other words, we assue a universality of the chemical freeze-out condition. This relationship between temperature and baryon chemical potential is very reasonable since all particle abundancies measured so far follow it. Once the width of the distribution of fireballs

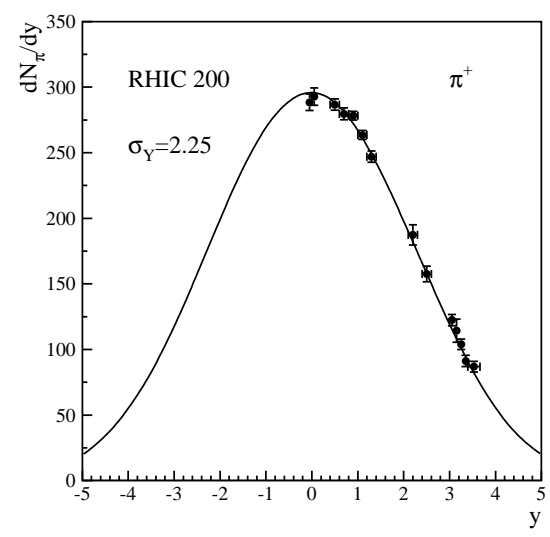

Figure 2. Fit to the pion distribution as measured by the BRAHMS collaboration.

has been fixed, we can go on with the dependence of the baryon chemical potential on the rapidity of the fireball (units are in $\mathrm{GeV}$ ).

$$
\mu_{B}=0.026+a y_{F B}^{2}
$$

The dependence of the $\bar{p} / p$ ratio on the parameter $a$ is shown in Figure [3]. The best value is $a=0.012 \mathrm{GeV}$.

The variation of the temperature along the rapidity axis is shown in Fig. 4. The temperature is maximal at mid-rapidity and gradually decreases towards higher (absolute) values of the rapidity. The baryon chemical potential follows a different pattern: it has a minimum at mid-rapidity and increases quite substantially towards higher values of the rapidity. Of particular interest are the largest values of the rapidity (say $y \approx 4$ ). It can be seen that there is a region of overlap with the data obtained at the NA49 experiment at CERN-SPS.

\section{Summary}

Particle yields measured in heavy ion collisions show an overwhelming evidence for chemical equilibrium at all beam energies. The rapidity dependence of the thermal parameters $T$ and $\mu_{B}$ can now be determined over a wide range of rapidities and show a systematic behavior towards an increase in $\mu_{B}$ as the rapidity is increased. 


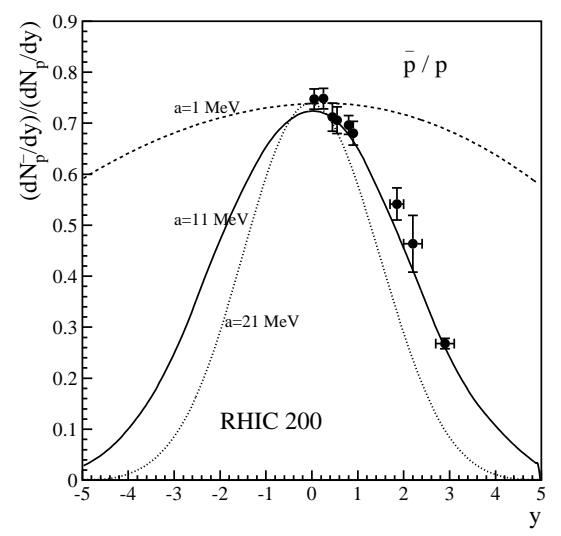

Figure 3. The $\bar{p} / p$ ratio as a function of rapidity. The curves show the dependence on the baryon chemical potential on the rapidity as discussed in the text.

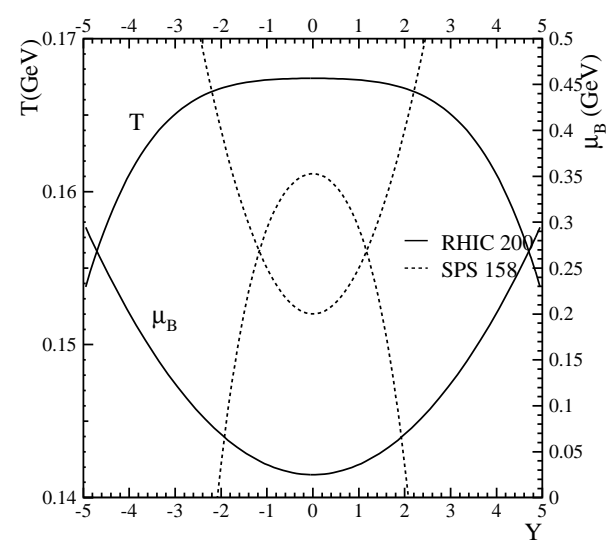

Figure 4. Values of the chemical freeze-out temperature and baryon chemical potential as a function of rapidity at the highest SPS (dotted lines) and RHIC (full lines) energies.

\section{Acknowledgments}

This work was supported in part by the Scientific and Technological Co-operation Programme between Italy and South Africa, project number 16. The help of D. Cebra with the preliminary $4 \pi$ data at the lower AGS beam energies is gratefully acknowledged, R. Adams has helped in the analysis of these data in Fig. [1] is acknowledged.

\section{References}

[1] For references see e.g. J. Cleymans, H. Oeschler, K. Redlich, S. Wheaton, Phys. Rev. C73 (2006) 034905.

[2] J. Cleymans, I. Kraus, H. Oeschler, K. Redlich, S. Wheaton, Phys. Rev. C74 (2006) 034903.

[3] J. Randrup and J. Cleymans, Phys. Rev. C74 (2006) 047901.

[4] L.A. Stiles and M. Murray/nucl-ex/0601039, unpublished. 
[5] D. Röhrich, Proceedings of the Talk given at the Workshop on "Critical Point and the Onset of Deconfinement", Florence, Italy, July 2006.

[6] I. Arsene at al., BRAHMS Collaboration, Phys. Rev. Lett. 94 (2005) 162301.

[7] I.G. Bearden, BRAHMS Collaboration, contribution to the QM2006 conference, Shanghai, China, November 2006.

[8] B. Biedron, W. Broniowski, nucl-th/0610083.

[9] F. Becattini and J. Cleymans (in preparation).

[10] W. Broniowski and F. Florkowski, Phys. Rev. C65 (2002) 064905; J. Phys. G31 (2005) S1087. 